\title{
La recepción del pensamiento de Michel Foucault y de Emmanuel Levinas en el proyecto de la Filosofía de la Liberación de Enrique Dussel.
}

\section{The Reception of the Thought of Michel Foucault and Emmanuel Levinas in the Project of the Philosophy of Liberation of Enrique Dussel.}

\section{A recepção do pensamento de Michel Foucault e Emmanuel Levinas no projeto da Filosofia da Libertação de Enrique Dussel.}

Mg. Luz María Lozano Suárez

Recibido: 23/11/2016 - Aceptado: 05/12/2016

\begin{abstract}
Resumen:
El punto de partida de Enrique Dussel es la víctima, el oprimido. La víctima tiene un lugar privilegiado en su Filosofía de la Liberación. Enrique Dussel retoma el concepto de Otro de Emmanuel Levinas para poder hablar de un sujeto-víctima. Sin embargo para Enrique Dussel es Foucault quien permite poner en evidencia una pluralidad de estructuras de dominación y numerosas víctimas ignoradas por el sistema que deben ser tratadas en la Ética de la Liberación. Enrique Dussel afirma que Foucault hace un análisis específico de los subsistemas y de las instituciones de exclusión. ¿Podríamos encontrar en la obra de Foucault nociones fundamentales para la construcción del concepto de sujetovíctima? ¿Sería apropiado relacionar el pensamiento foucaultiano y el levinasiano en la propuesta del filósofo latinoamericano? Analizando
\end{abstract}

1 Nacionalidad colombiana, licenciatura en Filosofía, maestra en arte dramático, magíster en educación, actualmente cursa un doctorado en filosofía en la Universidad Paris VIII y es profesora del departamento de Ciencias Humanas de la Universidad del Atlántico en Colombia. Contacto lumalosu@gmail.com 
la Filosofía de la Liberación de Enrique Dussel encontramos que estas dos filosofías francesas se hacen fecundas y se aventuran a puntos de encuentros para convertirse en pilares de la construcción de un proyecto cuyo centro es la víctima.

Palabras clave: Razón estratégica - sujeto-víctima - liberación - exclusión - poder.

\begin{abstract}
:
Enrique Dussel's starting point is the victim, the oppressed. The victim has a privileged position in his Philosophy of Liberation. Enrique Dussel takes up the concept of Other of Emmanuel Levinas to be able to talk about a subject-victim. However, for Enrique Dussel it is Foucault who makes it possible to highlight a plurality of structures of domination and numerous victims ignored by the system that must be addressed in the Ethics of Liberation. Enrique Dussel claims that Foucault makes a specific analysis of subsystems and exclusion institutions. Could we find in Foucault's work fundamental notions for the construction of the subject-victim concept? Would it be appropriate to relate Foucauldian and Levinasian thinking to the proposal of the Latin American philosopher? Analyzing the Philosophy of the Liberation of Enrique Dussel we find that these two French philosophies become fruitful and venture to meeting points to become pillars of the construction of a project whose center is the victim.
\end{abstract}

Keywords: Strategic reason - subject-victim - liberation - exclusion - power

\title{
Resumo:
}

O ponto de partida de Enrique Dussel é a vítima, o oprimido. A vítima tem um lugar especial em sua Filosofia da Libertação. Enrique Dussel retoma o conceito de Outro de Emmanuel Levinas para poder falar de um sujeito-vítima. No entanto, para Enrique Dussel é Foucault quem permite colocar em evidencia uma pluralidade de estruturas de dominação e numerosas vítimas ignoradas pelo sistema que devem ser abordadas na Ética da Libertação. Enrique Dussel argumenta que Foucault faz uma análise específica dos subsistemas e das instituições de exclusão. Poderíamos encontrar na obra de Foucault noções fundamentais para a construção do conceito de sujeito-vítima? Seria adequado relacionar o pensamento foucaultiano e levinasiano na proposta do filósofo latino-americano? Analisando a Filosofia da Libertação de Enrique Dussel encontramos que essas duas filosofias francesas tornam-se frutuosas e se aventuram a pontos de encontros para se converter em pilares da construção de um projeto cujo centro é a vítima.

Palavras-chave: Razão estratégica - sujeito-vítima - libertação - exclusão - poder. 
Este trabajo busca exponer de qué manera la recepción de las filosofías de Michel Foucault y de Emmanuel Levinas en América Latina han podido ayudar a construir una Ética y una Política de la Liberación, que tienen como eje principal una reflexión sobre las víctimas. Estas podrían ser visibilizadas y tratadas gracias a la concepción de sujeto en Levinas y al análisis de la dinámica poder-verdad-sujeto en que se enmarcó el trabajo de Foucault, pues estos análisis podrían dar cuenta de la opresión y de la exclusión como posibles formas de categorización de la victimización, tal y como lo hace notar Enrique Dussel. Por lo tanto trataremos de analizar aquí dos ejes primordiales del pensamiento que Dussel atribuye a Foucault y a Levinas en su Filosofía de la Liberación: la concepción del sujeto víctima de la Ética de la Liberación y la concepción de la razón estratégica instrumental para la construcción de una Política de la Liberación.

El pensamiento de Emmanuel Levinas es evidente en la Filosofía de la Liberación, pero ¿el pensamiento de Foucault puede estar articulado en el proyecto de Dussel? Foucault está alejado de una filosofía que llame a la liberación al estilo de Dussel. Como lo describe Linda Martin Alcoff (2000), Foucault de su lado hubiera podido sentir horror de la universalidad de las afirmaciones de Dussel, en la manera radical de declarar que sus ideas son válidas y en su proyecto de desarrollo y de defensa de normas éticas universales.

Partiendo del hecho que Foucault no tuvo la pretensión de concebir una teoría ética o política como posibilidad de hacer frente a los juegos de poder-verdad como lo es la filosofía de Enrique Dussel, y en cierta medida en Levinas al proponer la ética como filosofía primera, se asume este trabajo como un diálogo ente los tres autores. Foucault por su parte estuvo muy lejos de concebir una ley, teoría o fórmula que pudiera servir a los otros para pensar la libertad, que, sin embargo, es definida por él como la condición ontológica de la ética, y la ética a su vez, como la reflexión sobre la libertad. Dussel, de su lado, propone una ética y política que permitirían la libertad del pueblo latinoamericano. Por lo tanto este estudio puede ser una propuesta que queda abierta a desarrollarse, como una reflexión inminente y necesaria desde el momento en el que, desde la perspectiva de Fou- 
cault, podemos afirmar que la resistencia es el valor de la crítica y es, al mismo tiempo, la palabra clave de la dinámica poder-verdad-sujeto que permite la crítica y, como tal, la libertad.

Cabe anotar que en sus trabajos, Foucault se encarga de mostrar cómo la lucha de la liberación podría ser posible para el ejercicio de la libertad, pensada como la posibilidad de control de las relaciones de poder. La libertad para el filósofo francés, desde la propuesta de Enrique Dussel, no se consigue legalmente (libertad-contrato) ni es políticamente conquistada (liberación-conflicto) sino que, a cada instante, puede ser ejercida. Según Dussel, las prácticas de la libertad de las que nos habla Foucault son, en todo momento y en todo lugar, ejercidas aumentando o disminuyendo su propio margen de posibilidades. Es una libertad que evidentemente tiene un marco ético.

Es verdad que en la obra de Foucault podemos visionar una salida ética al problema de la sujeción que puede aparecer en la dinámica poder-sujeto-verdad. Sin embargo la salida podría ser totalmente individualista, entendida como trabajo estético de sí mismo y esta idea está totalmente alejada de la propuesta de Dussel. Frédéric Gros nos hace notar que la relación consigo mismo o cuidado de sí podría ser pensada como resistencia a los sistemas de poder, como forma de deshacer un poder caduco o modo de pasar de un poder a otro.

Enrique Dussel por lo tanto, al estar muy lejos de una pretensión al estilo foucaultiano, su intención desde el principio de sus investigaciones ha sido la de concebir una ética ontológica que pueda tener el carácter de validez universal. Y a pesar de que a partir de su trabajo de 1983, La dialéctica hegeliana, abandona la ética ontológica para dedicarse a la ética de la liberación (García 2014 782), seguirá concibiendo de todas formas a la ética como parte de la ontología porque es capaz de pensar en el obrar del hombre desde y fundado en el ser. El filósofo argentino se encargará de mostrar la debilidad del edificio de las éticas construidas sobre la metafísica de la subjetividad. Para Dussel era más positiva la construcción de una ética ontológica, una ethica perennis (Dussel 1972 155). Para Dussel la historia de la ética ontológica (ethica perennis) se ha ido fraguando en el oscuro hontanar 
de las éticas filosóficas dadas, que no fueron sino el pensar determinado a partir de êthos concretos. Dussel, por lo tanto, concibe que lo mismo es decir de-strucción de la historia de las éticas filosóficas, que des-cubrimiento de la gestación histórica de la ética ontológica. Luego asumió Dussel un proyecto de una ética que busca superar la modernidad, en el sentido trans-moderno en el que busca Dussel concebir una ética pensada desde Latinoamérica como exterioridad. Para Dussel, la metafísica del sujeto se concretó históricamente, desde el siglo XVI, como dialéctica del dominador-dominado. El proyecto será entonces la superación de la Modernidad como superación histórica, en un movimiento de liberación que sólo es posible a partir de la crítica de la subjetividad moderna, haciendo relevante la crítica de Levinas. Por lo tanto una crítica a la ética gestada desde el sujeto transcendental por ser una ética que no tiene en cuenta el carácter material: la vida concreta de cada individuo. Lo interesante para Dussel es poder pensar el nivel material de la ética de la liberación en lo que se refiere a la vida concreta de cada sujeto ético en comunidad. Por tal motivo advierte que el sentido de la ética propuesta será que vaya en miras de concebir una ética de contenido o material.

Dussel afirma que su ética parte de la tradición que niega al sujeto performativo que, siguiendo la crítica de los postmodernos, será la crítica a un sujeto derivado del Cogito cartesiano. Tal como afirma Dussel: "La negación del sujeto moderno es el horizonte desde donde se pudo afirmar un nuevo tipo de subjetividad" (Dussel 1998 515). Sin embargo la subjetividad para Dussel es comprendida primero en cuanto a su corporalidad, es el sujeto concreto, es vista la subjetividad de esta manera como un momento de la corporalidad humana, Dussel nos dice:

Cuando Descartes definió el "Yo pienso" (sujeto teórico autoconsciente) como el fundamento y esencia de la subjetividad, la de un alma a la que le es indiferente tener un cuerpo, produjo una falacia reductiva de las mayores consecuencias. Abrir la subjetividad más allá del sujeto, e implantada desde la corporalidad, fue el objeto de toda la filosofía francesa del siglo XX (Dussel 1999 3). 
Para Dussel por lo tanto el sujeto es un sujeto socio-histórico y su punto de partida excluye la concepción del sujeto moderno cartesiano para poder abordar de esta manera al sujeto concreto. Dussel se autodenomina posmoderno por su negación del sujeto moderno por considerarlo como un obstáculo epistemológico para concebir la víctima desde su corporalidad. Afirma que: "para Descartes el sujeto, el ego cogito, es un momento de un alma descorporalizada, cuya función es esencialmente cognitiva" (Dussel 1998 515).

Para demostrar la importancia de Foucault en una construcción del proyecto de liberación en Dussel, partiré de la siguiente afirmación que hace este filósofo sobre la idea del sujeto de su filosofía de la liberación:

El sujeto de la praxis de la liberación es el sujeto vivo, necesitado, natural, y por ello cultural, en último término la víctima, la comunidad de víctimas y los que a ella co-responsablemente articulados. El "lugar" último, del discurso, del enunciado crítico, son las víctimas empíricas, cuyas vidas están en riesgo, descubiertas en el diagrama del poder por la razón estratégica (Dussel 1998 525).

Para Dussel, Foucault se hace necesario en el proyecto de la Liberación puesto que una acción políticamente eficaz, desde un punto de vista estratégico, debe asumir un análisis del poder tal y como lo hizo Foucault.

Para que la intervención tenga un resultado de estabilización o transformación (sea cual fuere el objetivo estratégico) de la acción con la que intenta hacerse presente en el campo político, público entonces y resistente a toda intervención política que no logre doblegar, de alguna manera, el estado previo de las cosas (Dussel 2009 477).

Dussel, dentro de su proyecto de liberación, en lo que llama la etapa de la razón estratégica, el nivel práctico de factibilidad estratégica, asume un análisis del poder tal y como es descrito por Foucault para poder hablar del aspecto material en la Ética de Liberación, ya que las relaciones del poder descritas por Foucault permitirían develar la 
exclusión. Es de especial interés analizar de qué manera el trabajo de los excluidos que subyace en la analítica foucaultiana puede dar cuenta de la victimización, tal y como la plantea Enrique Dussel.

Para Dussel, la razón estratégica se sitúa en el nivel de la microfísica del poder. Nos dice que:

La Ética de la Liberación tiene mucho que aprender de Foucault en su análisis dentro del horizonte de la factibilidad crítico-práctica de subsistemas o de "instituciones" históricas. Por ello, y no abandonando tampoco la macrofísica del poder, la razón estratégica, que maneja las relaciones de Poder, queda delimitada en el marco de la posibilidad material (Dussel 1998 499).

Según Dussel el poder es tomado en sentido foucaultiano porque permite una dinámica de libertad:

Ejercita poder el que modifica la red, y los puntos o nudos de la red (y por ello campo minado, ya que cada voluntad en la estructura del poder es una posible mina, una posible explosión, un conflicto inevitable), o el campo magnético del poder. No necesariamente domina otras voluntades, ya que el que pasivamente resistía puede pasar a un estado de igualdad sin ejercer dominio (pero sin dejarse ya dominar), y eso es ya un nuevo ejercicio del poder. Puede establecerse en la igualdad; puede intentar pasar a la hegemonía; puede caer en la pasividad resistente. Todo es posible, pero se manifiesta como poder al modificar la estructura. Pero aún al no modificarla se puede seguir ejerciendo un poder, al menos como resistencia, y en ciertos casos como quitando la posibilidad del ejercicio de la fuerza al hacer el vacío (Dussel 2009 476).

Dussel nos dice que Foucault muestra la articulación entre este saber disciplinario -en el hospital, prisión, escuela, hogar, cuartel, tribunales, administración, fábrica, etc.- que prepara los cuerpos para el cumplimiento de sus funciones en la dispersa y plural distribución del poder en el campo de la microfísica de los subsistemas institucionales. 
El interés que tiene Dussel por Foucault se centra principalmente en lo que Foucault podría aportar en el nivel material de la Ética de la Liberación porque para Dussel el orden del poder, de las relaciones de fuerza y de los cuerpos es material.

Lo interesante para Dussel es poder pensar el nivel material de la Ética de la Liberación en lo que se refiere a la vida concreta de cada sujeto ético en comunidad. Por tal motivo advierte que el sentido de la ética propuesta será que vaya en miras de concebir una ética material.

Para Dussel, Foucault hace posible situarse en la razón estratégica a través del tipo de análisis del poder que permite evidenciar las víctimas. La forma de poder (de la) que según Foucault se ejerce sobre la vida cotidiana de los individuos, designándoles su lugar y su identidad a través de ciertos códigos que ellos deben reconocer y deben reconocer en los otros en un momento histórico determinado. Esta es la forma de poder que transforma a los individuos en sujetos.

Dussel observa el tema de la vida en el cuadro de la biopolítica planteado por Foucault como punto de partida para entender lo que podría ser la cuestión de la corporalidad de los excluidos. Dussel plantea que Foucault habla del bio-poder, de la bio-política, de la gestión de la vida como lo propio de las relaciones de fuerza en las que consiste el poder. Llama la atención a Dussel del análisis de Foucault, el cómo se ha vuelto un objetivo la vida en el juego de las luchas políticas: El derecho a la vida, al cuerpo, a la salud, a la felicidad, a la satisfacción de las necesidades.

Para Dussel esto permite una fundamentación de una ética que parte de: a) el criterio de verdad práctica o del contenido de toda ética; b) principio material y universal de la ética, y c) la problemática de la "aplicación" del principio ético material (Dussel, 1998: 130), cuestión que se desarrollará a fondo para poder comprender el momento material de la ética en el que vincula Dussel a Foucault. Según Dussel, Foucault pasa del orden formal del lenguaje al orden material (el nivel de la producción, reproducción y desarrollo de la vida corporal humana de cada sujeto ético). 
El poder entonces para Dussel toma la definición de una relación de fuerzas. Para Dussel, las fuerzas consisten en acciones en tensión sobre otras acciones, que se auto-sostienen en la gestión de la vida.

Dussel quiere por lo tanto llegar a subsumir el análisis de Foucault desde una razón estratégica porque considera que es importante para la Ética de la Liberación en cuanto propone la necesidad de un sujeto histórico. De esta manera Dussel pudo colocar la corporalidad de los excluidos y la vida de los sujetos como última instancia de la Ética de la Liberación.

De lo que estamos hablando entonces es de la vida de cada sujeto, última referencia activa, como organismo autorregulado viviente, social e histórico, pero igualmente como crítico autoconsciente, sin reduccionismo (como cuando se pretende definir como lo esencial al sujeto como cogito), pero tampoco con unilateralismos (como cuando se niega a todo sujeto, al Cogito o al sujeto transcendental kantiano con todo tipo de subjetividad) (Dussel 1998 524).

Hay que aclarar que al momento de hablar de exclusión, Dussel no se refiere a la opresión. Deja claro en su Ética de la Liberación que se trata de la alteridad de la víctima como oprimida o excluida. Anotemos que para Dussel la exterioridad de la exclusión no es idéntica a la exterioridad de la opresión.

La Ética de la Liberación pretende abarcar de igual manera dos ámbitos de la victimización enmarcada siempre como una exterioridad frente a la totalidad, tomada obviamente en sentido levinasiano. Es por ello menester anotar que, aunque Dussel tome el trabajo de Foucault como el análisis de subsitemas o instituciones de exclusión, el filósofo argentino no se separa del concepto de alteridad de Levinas.

Es de suma importancia señalar que resulta bastante raro poder encontrar la referencia de Foucault en la obra de Enrique Dussel, puesto que el filósofo argentino toma como punto de partida para la construcción de su ética a las víctimas o sujeto víctima desde la condición de subjetividad desarrollada por Emmanuel Levinas, quien le permite 
hablar de la alteridad. Evidentemente para concebir la cuestión del sujeto como víctima, Dussel ha tomado como punto de apoyo el pensamiento de Levinas. Para Foucault el tema de la alteridad no fue de su interés. Dussel nos dice:

Nuestro mundo no ha sido sólo pedagógicamente abierto desde el Otro; nuestro mundo queda esencialmente abierto desde la Alteridad, es alternativo por su propia naturaleza. El otro es el origen primero y el destinatario último de todo nuestro ser-en-el-mundo. El cara-a-cara es la experiencia primera, radical de nuestro ser de hombres. Es el habérselas de manera supremamente real con el ser; no ya en el modo de la com-prensión del ser, sino como la apertura y exposición meta-física o ética ante el rostro como límite después del cual el Otro, personal, se levanta como quien teniendo derecho, exige justicia, propone la paz y protesta ante mi pretensión totalizante (totalitaria) de comprenderlo. Debemos entonces describir el carácter incom-prensible de la presencia del Otro; el "Otro permanece infinitamente transcendente, infinitamente extranjero, y como rostro, donde se produce su epifanía y desde donde me llama, rompe con el mundo que pudiera sernos común (Dussel 1987 123).

Para Dussel el referente privilegiado es la víctima, que operará como el sujeto en última instancia. El sujeto es definido como un sujeto vivo, necesitado, natural, y por ello cultural, en último término la víctima, la comunidad de las víctimas y de los que a ella co-rresponde articulados. El lugar último, entonces, del discurso, del enunciado crítico, son las víctimas empíricas, cuyas vidas están en riesgo, descubiertas en el diagrama del Poder por la razón estratégica.

La preocupación a la que se dirige la propuesta de Enrique Dussel es la víctima, a la que dará la categoría de "sujeto" en la praxis de la liberación. Las víctimas tienen un lugar privilegiado desde el desarrollo del discurso de Dussel.

Cada sujeto ético de la vida cotidiana, cada individuo concreto en su modo de actuar, es ya un sujeto posible de la 
praxis de la liberación, en cuanto víctima o solidario con la víctima, fundamente normas, realice acciones, organice instituciones o transforme sistemas de eticidad... sin embargo, lo propio de esta ética o su referente privilegiado es la víctima o comunidad de víctimas, que opera como el/los sujetos en última instancia" (Dussel 1998 513).

En última instancia es el sujeto víctima, como sujeto material negado por el sistema.

Es desde este "irse muriendo del sujeto real", del soporte material de la corporalidad (y no como una mera y metafórica "muerte del sujeto" filosófico virtual) bajo el papel del "actor funcional" y alienado en el sistema, que el sujeto concreto (cuya subjetividad doliente le hace presente su propia corporalidad concreta vulnerable y lacerada, que no puede vivir)" (Dussel 1999 16).

Su perspectiva es tomada de la visión del sujeto desde Levinas. "En Levinas, la negación del sujeto es ética, puesto que ella opera en nombre mismo de la ética, y por unas razones explícitamente éticas, es decir, morales." (Misrahi 1994 175)²

Pareciera así que las víctimas adquieren un criterio Absoluto que para Dussel es definido como el "sujeto corporal vivo". Es la vida de las víctimas mostrada como dignidad, el Absoluto identificado en la praxis de la liberación. Es lo Absolutamente otro que toma de Levinas para concretizarlo en las víctimas.

Desde Levinas se delimita la concepción de sujeto desde la ética tal y como sigue:

Descubrir del yo una tal orientación, es identificar Yo y moralidad. El Yo antes delante del Otro, es infinitamente

2 La négation du sujet est éthique par elle-même, puisqu'elle s'opère au nom même de l'éthique, et pour des raisons explicitement éthiques, c'est-à-dire ici, morales. (Traducción del francés, la autora). 
responsable. El otro que provoca ese movimiento ético en la conciencia, que perturba la buena conciencia de la coincidencia misma con él mismo... incremento de exigencias con respecto de sí: más hago frente a mis responsabilidades y más yo soy responsable. (Levinas 197254$)^{3}$.

Sin embargo, según Dussel, Levinas habla siempre de que el otro es "absolutamente otro". Tiende entonces hacia la equivocidad. Por otra parte, nunca ha pensado que el otro pudiera ser un indio, un africano, un asiático. El otro para nosotros, es América Latina con respecto a la totalidad europea; es el pueblo pobre y oprimido con respecto a las oligarquías dominadoras (Dussel 1973 113). Es por ello que al hablar de un sujeto concreto se va luego hacia la propuesta de Foucault.

Dussel, de esta manera, permite el encuentro de Levinas y Foucault en su proyecto de liberación, quienes, aunque sean filósofos tan diferentes, permiten pensar la necesidad de la ética para hacer frente a las acciones de sujeción. Sin embargo, los caminos son dispares. Para Foucault y Levinas era necesaria una reflexión ética que pudiera hacernos pensar al sujeto de otro modo, de tal manera que se diera la posibilidad de responder a los abusos de la dominación-totalización. En cuanto a la subjetividad, Foucault buscará la de-subjetivación y Levinas parte de la concepción de la subjetividad para concebir la alteridad. "Es Levinas el filósofo que permite a Dussel protagonizar una ruptura teórica fundamental principalmente a través de la recepción que nuestro filósofo hace de las categorías de otro y exterioridad, categorías con las que supera el sentido totalizador del mundo heideggeriano." (González 2014 50).

Para Levinas la ética se constituye como la filosofía primera, para Foucault la vida filosófica está relacionada con el trabajo ético que

3 Découvrir au Moi une telle orientation, c'est identifier Moi et moralité. Le Moi devant Autrui, est infiniment responsable. L'autre qui provoque ce mouvement éthique dans la conscience, qui dérègle la bonne conscience de la coïncidence même avec lui-même... Accroissement d'exigences à l'égard de soi: plus je fais face à mes responsabilités et plus je suis responsable. (Traducción del francés la autora). 
un individuo realiza sobre sí mismo. Levinas retoma su propuesta desde la lectura de la Biblia y Foucault lo hará inspirándose en las éticas de la antigüedad greco-romana. Es importante resaltar que en ambos hay una necesidad de la ética como respuesta, como resistencia, y no una teoría política. Levinas para Foucault habría sido un retorno a las prácticas de obediencia tal y como lo hace con su crítica a la pastoral cristiana. El trabajo de Foucault para Levinas sería una filosofía que va en camino del egoísmo o del individualismo. Resaltamos que no es el interés de este trabajo entrar en esta problemática. Lo que sí resulta interesante es el observar cómo es posible que Enrique Dussel haya podido establecer dentro de su Ética de la Liberación dos filosofías tan diferentes. ¿Cómo es posible que confluyan dos pensamientos de corte tan diferente en la Ética de la Liberación de Dussel para construir su significado de Sujeto víctima? ¿Existe acaso un punto en común entre Levinas y Foucault? ¿Cómo es posible que dos corrientes filosóficas puedan tener confluencia en una propuesta filosófica?

Por otro lado, dentro del desarrollo del trabajo de Foucault sobre su análisis del poder-sujeto-verdad parece no haber cabida para hablar de víctimas, pues dentro de la relación poder-sujeto-verdad puede bosquejarse la exclusión, pero no una victimización en cuanto tal. Recordemos que el análisis que hace Foucault del poder no es en términos negativos, simplemente, el poder produce, él produce realidades. El individuo y el conocimiento revelan esta producción (Foucault 1975).

Es evidente que Foucault no hace referencia a un sujeto concerniente a la víctima o a una comunidad de víctimas, pero tampoco se podría afirmar que un análisis de las víctimas sería totalmente excluido porque este podría ser concebido en su concepción del poder, el cual puede caer en la dominación. Aunque no se tenga en cuenta un poder opresivo o negativo, sino un poder que produce sujetos, este poder podría dar lugar a victimización. En Foucault también hay la intención de sugerir un tipo de poder negativo. Recordemos que Foucault habla de relaciones de poder que son fijas o de dominación. Estas son las relaciones de poder, que en lugar de ser 
móviles y abiertas a la modificación, se encuentran bloqueadas o fijas (Foucault 1984 1530).

Para la construcción de una Ética de la Liberación, Dussel acude entonces sin miramientos a Foucault para poder identificar a las víctimas a partir del análisis que permite dar cuenta de la exclusión.

La crítica de Foucault, desde "los excluidos de la sociedad", nos permite descubrir muchas estructuras de dominación, numerosas víctimas ignoradas, que deberán ser tratadas particularmente en otro momento de la Ética de la Liberación -cuando expongamos los numerosos "Frentes de liberación" en las luchas por el reconocimiento (Dussel 1998 452).

Maurice Blanchot (2002) nos hace notar que el trabajo que hizo Foucault sobre la locura, no fue un trabajo de la locura directamente. La locura fue un pretexto para entrar en el ámbito de la exclusión. Foucault hizo un trabajo sobre el poder de exclusión. De esta manera el filósofo francés podría mostrar cómo se había construido todo un saber subsumido por el aparato administrativo alrededor de lo razonable y lo irrazonable para poder discriminar lo que podría ser peligroso y tomar los correctivos necesarios dentro de una sociedad de Ortopedia.

El mismo Foucault en entrevista con Claude Bonnefoy, publicada en 2011, nos dice que él nunca se planteó el problema de la relación obra-enfermo, sino de la relación exclusión-inclusión: exclusión del individuo, de sus gestos, de su comportamiento, de su carácter.

El problema es el de establecer de qué manera podríamos categorizar a los excluidos desde el trabajo realizado por Foucault. En la constitución de sujetos en la dinámica de los juegos de poder-verdad, se encuentra ese tipo de sujeto no incluido dentro de ese sistema que permite rechazar la diferencia y puede llegarse a pensar que es un sujeto descategorizado, que no puede ser nombrado. Por ejemplo, Foucault se refiere a la exclusión del otro citado por $\mathrm{H}$. Deyfrus y $\mathrm{P}$. Ravinow, cuando habla de la sociedad griega que estaba relacionada 
con una sociedad viril; esta es la idea de disimetría, de exclusión del otro (Deyfrus y Ravinow 1984 327).

Tal y como nos lo expresa Sichère (1990), en muchas comunidades, ciertos individuos son excluidos de la posibilidad de constituirse en sujetos. Es así como el trabajo sobre el sujeto en Foucault nos ha dado la posibilidad de excluir otro tipo de sujeto que no aparece en el horizonte de las prácticas humanitarias que buscan la libertad de los individuos en la actualidad, pues para Foucault hay otras formas de libertad posibles más allá del sueño humanista, porque este igualmente devela exclusión.

Es bien sabida la dificultad de poder nombrar a un sujeto categorizado como víctima desde el análisis foucaultiano. El problema es cómo surge un lenguaje regular y normativo que pueda decir quién es el excluido. Desde la Ética de la Liberación que propone Enrique Dussel vale la pena el trabajo de Foucault para poder analizar entonces cómo han sido desarrollados los dispositivos de exclusión en América Latina y de esta manera dar cuenta de las víctimas. Hemos tomado la propuesta de la Ética de la Liberación de Enrique Dussel siguiendo las pistas a su proyecto que aspira a permitir una ética y política eficaces que puedan evitar la dominación y asumir la resistencia en sentido foucaultiano, y, de esta manera, observar que el trabajo de Foucault se puede pensar como posibilidad de desarrollo de una ética y política que puedan partir su análisis del poder-sujeto-verdad; puesto que según Dussel, la estructura del poder puede ser modificada y para eso asume en su proyecto el análisis del poder según el filósofo francés.

Finalmente podemos decir que el sujeto aparece en el sistema como Otro. El Otro, que es tomado por Dussel de la idea de sujeto en Levinas, es la vida negada de la víctima. Para Dussel, el último lugar del enunciado crítico es la víctima empírica, entendiendo de forma concreta al sujeto.

Dussel distingue distintos niveles en los que se muestra lo fructífero de la concepción levinasiana de la alteridad cuando se le comprende con las adecuadas mediaciones: a nivel mundial (centro-periferia), a nivel nacional (élites-masas, 
burguesía nacional-pueblo), a nivel erótico (mujer-varón), a nivel pedagógico (cultura elitista-cultura popular), a nivel religioso (el problema del fetichismo). El otro, absolutamente otro, de Levinas es concretizado por medio de la analogía: el otro es el indio empobrecido, el negro segregado, el judío exterminado, el africano y asiático discriminado, la mujer como objeto sexual, el niño o el joven manipulado por una educación ideológica, etc. (García, Pedro 2014 784).

Estas víctimas son descubiertas por el diagrama del poder gracias a la razón estratégica. De esta manera el análisis del poder concebido por Foucault permitirá a Dussel descubrir las víctimas porque, según Foucault, el poder es ejercido sobre la vida de individuos para producir sujetos y dentro de estos sujetos un tipo de subjetividad sería la víctima. Dussel se sirve de Foucault para identificar las víctimas en la microfísica del poder y así poder visibilizarlas. La cuestión del sujeto víctima en Dussel nos lleva a hablar de la exigencia de justicia. Para Dussel se trata de utilizar la concepción del poder foucaultiana a partir de la razón estratégica para identificar las víctimas que en un primer momento fueron definidas por Levinas.

\section{Bibliografía}

Blanchot, Maurice. Michel Foucault tel je l'imagine. Paris: Gallimard, 2002.

Deyfrus, H. y Ravinow, P. Michel Foucault, un Parcours philosophique. Paris: Editions Gallimard, 1984.

Dussel, Enrique. Para una de-strucción de la historia de la ética. Mendoza: Facultad de filosofía y letras de la Universidad Nacional de Cuyo, Instituto de filosofía, 1972.

Dussel, Enrique. América Latina: dependencia y Liberación. Buenos Aires: Editorial Fernando García Cambeiro. 1973.

Dussel, Enrique. Filosofía ética de la liberación Tomo I: presupuestos de una filosofía de la liberación. Buenos Aires: Asociación ediciones La Aurora, 1987.

Dussel, Enrique. Ética de la liberación en la edad de la globalización y la exclusión, 2 edición. Madrid: Editorial Trotta, 1998. 
Dussel, Enrique. "Sobre el sujeto y la intersubjetividad: el agente histórico como actor en los movimientos sociales". Revista Pasos 84 (1999).

Dussel, Enrique. Política de la Liberación II: Arquitectónica. Madrid: Editorial Trotta, 2009.

Foucault, Michel. Surveiller et punir. Paris: Editions Gallimard, 1975.

Foucault, Michel. L'éthique de souci de soi comme pratique de la liberté, en Dits et Écrits, vol II. Paris: Quarto Gallimard, 1984.

Foucault, Michel. Le beau danger. Entretien avec Claude Bonnefoy. Paris: Editions de l'Ecole des Hautes Études en Sciences Sociales, 2011.

García, Pedro. "Geopolítica de la alteridad. Levinas y la filosofía de la liberación de E. Dussel". ISEGORIA, Revista de Filosofía moral y política. Número 51 (2014): 777-792.

González, Patricia. "La filosofía de la liberación de Enrique Dussel. Una aproximación a partir de la formulación de la analéctica". Estudios de Filosofía Práctica e Historia de las Ideas. Vol. 16 nº 2 (2014).

Gros, Frédéric. Que sais-je?: Michel Foucault. Paris: Presses Universitaires de France, 1996.

Levinas, Emmanuel. Humanisme de l'autre homme. Paris: Fata Morgana, 1972.

Martín Alcoff, Linda. "Power/Knowledges in the Colonial Unconscious: A Dialogue between Dussel and Foucault". Thinking from the underside of history: Enrique Dussel's Philosophy of liberation. Martín Alcoff, Linda and Mendieta, Eduardo, editores. Boston: Rowman \& Littlefield Publishers, 2000.

Misrahi, Robert. La problématique du sujet aujourd'hui. Fougères: Encre Marine, 1994.

Sichere, Bernard. Eloge du sujet: Du retard de la pensée sur les corps. Paris: Editions Grasset \& Fasquelle, 1900. 